\title{
Clinical Study of Aspirin in the Prevention of Thrombosis in Breast Cancer Patients with Postoperative Chemotherapy after PICC Insertion
}

\author{
Cong Zhang1,2, Xiaofang Xiong1,2, Shihong Deng'2, Fang Wang1,2* \\ ${ }^{1}$ Department of Cardio-Thoracic and Breast Surgery, The First Affiliated Hospital of Yangtze University, Jingzhou, China \\ ${ }^{2}$ Team of Intravenous Therapy, The First Affiliated Hospital of Yangtze University, Jingzhou, China \\ Email: 11199926@qq.com, *1284843024@qq.com
}

How to cite this paper: Zhang, C., Xiong, X.F., Deng, S.H. and Wang, F. (2018) Clinical Study of Aspirin in the Prevention of Thrombosis in Breast Cancer Patients with Postoperative Chemotherapy after PICC Insertion. Yangtze Medicine, 2, 255-261. https://doi.org/10.4236/ym.2018.24027

Received: August 1, 2018

Accepted: December 18, 2018

Published: December 21, 2018

Copyright $\odot 2018$ by authors and Scientific Research Publishing Inc. This work is licensed under the Creative Commons Attribution International License (CC BY 4.0).

http://creativecommons.org/licenses/by/4.0/

(c) (i) Open Access

\begin{abstract}
Objective: To study the clinical effect of aspirin in the prevention of venous thrombosis in breast cancer patients with postoperative chemotherapy after peripherally inserted central catheter (PICC) insertion. Methods: 240 cases of female breast cancer patients with postoperative chemotherapy after PICC insertion in The First People's Hospital of Jingzhou from June 2014 to December 2017 were selected and divided into experimental group $(n=120)$ and control group $(n=120)$ according to the length of stay. The modified Seldinger technique was used in both groups. The experimental group had oral Aspirin enteric-coated tablet on the day of PICC insertion, $100 \mathrm{mg} /$ day until the PICC catheter was removed, while the control group did not take anticoagulant drugs. The therapeutic effects were evaluated by color Doppler ultrasound, Coagulation analysis, and complete blood count. Results: There were three cases with venous thrombosis in the experimental group, and the incidence rate was $2.5 \%$, while ten cases in the control group developed venous thrombosis, and the incidence rate was $10 \%$. There was a statistically significant difference in the incidence of thrombosis between the two groups ( $\mathrm{P}$ $<0.05)$. Conclusion: This study showed that oral Aspirin can effectively reduce the incidence rate of venous thrombosis in breast cancer patients with postoperative chemotherapy after PICC insertion. Therefore, it is worthy of clinical application.
\end{abstract}

\section{Keywords}

Aspirin, Breast Cancer, Peripherally Inserted Central Catheter, Venous Thrombosis 


\section{Introduction}

Currently, the multi-cycle adjuvant chemotherapy has become the most important treatment for breast cancer surgery, and peripherally inserted central catheter (PICC) has been widely used in the chemotherapy of malignant tumors [1]. Because patients after breast cancer surgery usually have edema in their affected limbs, which is caused by Lymphatic reflux obstruction, the clinical treatment of infusion and blood collection of the affected limbs is avoided. Therefore, breast cancer patients with chemotherapy have higher requirements for care after PICC insertion compared with other patients. As 6 - 8 cycles of adjuvant chemotherapy is mostly used in breast cancer patients, long-term indwelling PICC can induce venous thrombosis. It has been reported in the literature that the incidence of venous thrombosis can be as high as $38 \%$ after long-term indwelling PICC and even the severe venous thrombosis can be a life-threatening [2]. Therefore, it is essential to prevent the formation of thrombosis after PICC insertion. At present, it is inconclusive whether the preventive anticoagulant therapy can prevent PICC-related upper extremity venous thrombosis. Some foreign scholars have found that no use of any form of anticoagulant or antiplatelet drugs is mildly correlated with PICC-related thrombosis risk (odds ratio 1.93, $\mathrm{P}=0.036$, $95 \%$ confidence interval, 1.025 - 3.602) [3]. Findings in another study suggest that the preventive use of anticoagulant drugs can reduce the incidence of venous thrombosis in patients with PICC [4]. In this study, patients with PICC were given small doses of Aspirin tablets to observe the preventive effects on thrombosis after the insertion of PICC.

\section{Material and Methods}

\subsection{General Data}

A total of 240 female breast cancer patients in The First People's Hospital of Jingzhou from June 2014 to December 2017 were selected, aged 32 - 57, with a median age of 45 years old. Inclusion criteria: modified radical surgery for breast cancer; postoperative pathological diagnosis of breast cancer; pathological stage I-II; postoperative 6 - 8 cycles of adjuvant chemotherapy; previous history of no digestive ulcer; coagulation analysis result was normal; PICC insertion informed consent was signed; PICC insertion was successful, and the chest radiograph confirmed the catheter insertion site was normal. Exclusion criteria: infections occurred at insertion site during PICC insertion; accidental or artificially induced catheter shedding; there was bleeding tendency during anticoagulation and allergic reactions could not be tolerated.

\subsection{Method}

All patients were divided into experimental group $(n=120$, June 2014-December $2015)$ and control group $(\mathrm{n}=120$, January 2016-December 2017). There was no significant difference in the general data between the two groups $(\mathrm{P}>0.05)$. See Table 1 for details. Both groups were given catheter insertion by professionally 
Table 1. General data comparison between experimental group and control group.

\begin{tabular}{ccccccc}
\hline \multirow{2}{*}{ Group } & \multirow{2}{*}{$\begin{array}{c}\text { Number } \\
\text { of cases }\end{array}$} & \multicolumn{2}{c}{ Age } & \multicolumn{3}{c}{ Chemotherapy scheme } \\
\cline { 3 - 7 } & $30-45$ & $45-60$ & CEF & TEC & EC-T \\
\hline Experimental group & 120 & 64 & 56 & 38 & 54 & 28 \\
Control group & 120 & 62 & 58 & 35 & 60 & 25 \\
$\chi^{2}$ & & 0.067 & 0.067 & 0.177 & 0.602 & 0.218 \\
$P$ & & 0.796 & 0.796 & 0.674 & 0.438 & 0.641 \\
\hline
\end{tabular}

Note: CEF is cyclophosphamide + epirubicin + fluorouracil, TEC is docetaxel + epirubicin + cyclophosphamide, EC-T is epirubicin + cyclophosphamide sequential docetaxel Match.

trained nurses who have obtained PICC insertion qualifications, and the modified Seldinger technique was used in both groups to improve success rate and reduce complications [5]. A chest X-ray was taken after catheter insertion to confirm the site of the catheter tip and daily care was performed in accordance with the uniform standard: 1) change the dressing once every 7 days, keep local part clean, and the dressing should be changed immediately if it is loose or contaminated; 2) replace the heparin cap once every 7 days; 3) flush the catheter and keep the catheter smooth, once every 7 days during the treatment interval. The heparin dilution was used to seal the catheter during the insertion. The experimental group had oral Aspirin enteric-coated tablet on the day of the insertion, $100 \mathrm{mg} /$ day until the PICC was removed, while the control group did not take anticoagulant drugs.

\subsection{Evaluation Indicator}

Both groups of patients underwent color Doppler ultrasound on $3^{\text {rd }}$ day, $7^{\text {th }}$ day, $15^{\text {th }}$ day, $30^{\text {th }}$ day after PICC insertion and before extubation or when the patient developed redness, soreness and pain in the limb of insertion, so as to confirm the presence of venous thrombosis. Coagulation analysis and complete blood count were performed every 7 days to learn about the patient's blood changes and coagulation function.

\subsection{Statistical Method}

Statistical analysis was performed by using SPSS19.0 software. The count data were analyzed by $\chi^{2}$ test. $\mathrm{P}<0.05$ indicated that the difference was statistically significant.

\section{Results}

In both groups, except for some patients whose hemoglobin and thrombocytopenia were observed to be reduced during chemotherapy, considering the myelosuppression caused by chemotherapy drugs, the situation was improved after the subcutaneous injection on the patient with leukocyte drug. The experimental group showed no obvious skin bruising, bleeding tendency such as gum bleeding and Aspirin allergic reaction, and the coagulation analysis was normal. There 
were three cases with venous thrombosis in the experimental group, and the incidence rate was $2.5 \%$, including one case on the $5^{\text {th }}$ day after the insertion, one case on the $12^{\text {th }}$ day after the insertion, one case on the $27^{\text {th }}$ day after the insertion, while ten cases in the control group developed venous thrombosis, and the incidence rate was $10 \%$, including four cases occurred within 7 days after the insertion, three cases occurred within 7 - 15 days after the insertion, three cases occurred within 15 - 30 days after the insertion, and two cases occurred on the $48^{\text {th }}$ day after the insertion. Consequently, the incidence of thrombosis on both groups was statistically different $(\mathrm{P}<0.05)$. The incidence of venous thrombosis and the time of occurrence of the two groups of patients are shown in Table 2.

\section{Discussion}

At present, breast cancer has become the most common cancer in women, and the postoperative chemotherapy for breast cancer is mostly a chemotherapy regimen by focusing on using a multi-cycle anthracyclines. Since anthracyclines are foaming agents, once extravasation occurred, it can cause redness, blistering, hardening and severe necrosis of surrounding tissues, resulting in the serious consequences [6]. Therefore, PICC insertion or implantable intravenous infusion is mainly used in clinical practice to provide patients with relatively safe infusion channels. However, due to the hypercoagulable state of blood of cancer patients, studies have shown that patients with cancer have a higher risk of thrombosis embolism, and the incidence rate can reach 5\%, which is an increasing trend year by year [7]. But the risk of venous thrombosis in patients with breast cancer is five times to that of normal people especially those patients that have undertaken standardized chemotherapy are higher than the former [8]. Thus, while maintaining effective and safe venous access, PICC insertion has had the biggest complication of venous thrombosis. Especially in the long-term insertion, the risk of thrombosis increases due to the change of blood flow status at the catheter site. Therefore, the prevention of the formation of venous thrombosis and reduction of incidence of venous thrombosis after PICC plays a decisive role in the patient's condition and quality of life.

There are three main reasons for the formation of venous thrombosis in breast cancer patients with PICC: First is the hypercoagulable state of the blood: tumor cells can directly or indirectly activate the coagulation system in the body

Table 2. Venous thrombosis occurrence time and incidence of experimental group and control group.

\begin{tabular}{|c|c|c|c|c|c|c|c|}
\hline \multirow{2}{*}{ Group } & \multirow{2}{*}{$\begin{array}{l}\text { Number } \\
\text { of cases }\end{array}$} & \multicolumn{5}{|c|}{ Occurrence time of thrombosis } & \multirow{2}{*}{$\begin{array}{l}\text { Incidence rate } \\
\text { of thrombosis }\end{array}$} \\
\hline & & $3 \mathrm{~d}$ & $3-7 d$ & $7-15 d$ & $15-30 d$ & $>30 \mathrm{~d}$ & \\
\hline Experimental group & 120 & 0 & 1 & 1 & 1 & 0 & $2.5 \%$ \\
\hline Control group & 120 & 0 & 4 & 3 & 3 & 2 & $10 \%$ \\
\hline$\chi^{2}$ & & & & & & & 5.760 \\
\hline $\mathrm{P}$ & & & & & & & 0.016 \\
\hline
\end{tabular}


to produce thrombin by synthesizing various procoagulant substances. In addition, it can also secrete higher fibrinolytic inhibitors to inhibit the body's fibrinolytic activity, and produce platelet membrane glycoprotein and other substances that lead to platelet aggregation, while the anti-thrombin and anticoagulant system activity of cancer patients is significantly lower than normal people. Second, the damage of the blood vessel wall: the growth of tumor cells can directly infiltrate or compress the blood vessels, resulting in damage of vascular endothelial cells, releasing various cytokines to damage the vascular endothelium. Surgery, venipuncture, chemotherapy, and interventional therapy can also cause direct or indirect toxic effects on the vascular endothelium, thereby leading to the body in a state of thrombosis. Third is the venous blood flow stasis state: the tumor itself or the metastatic lymph nodes of the metastasis on the blood vessels results in slow local blood flow and formation of eddy current, and limb muscle relaxation causes blood flow stagnation due to the patient's long-term bed rest, thereby making the blood activated clotting factor clearance slowed down, endothelial cells impaired by hypoxia, platelets activated and aggregate and blood viscosity increase, which is prone to venous thrombosis [9].

For the prevention of venous thrombosis, the Guide of American Society of Clinical Oncology for the Prevention and Treatment of Venous Thromboembolism in Cancer Patients recommended the conventional prescription of anticoagulant drugs to prevent the formation of venous thrombosis for patients with cancer and without bleeding or other anticoagulation contraindications [10]. At present, clinically oral warfarin is used and the patient's INR level is adjusted between 1.5 and 2.5 to prevent the risk of the thrombosis after long-term insertion. However, there is a significant risk of bleeding in the use of warfarin, and patients need to repeatedly perform coagulation analysis and monitoring to adjust the dosage, the process is cumbersome with poor patient compliance. Aspirin enteric-coated tablets are a kind of the non-steroidal anti-inflammatory drug produced by Bayer AG. Because of its inhibitory effect on platelet aggregation, it is clinically used to prevent the formation of thrombosis. Some foreign scholars believe that warfarin and aspirin are with the similar effect in indwelling venous catheter patency in the long-term [11] [12]. The studies conducted by Tufano et al. have shown that after patients with platelet dysfunction have oral Aspirin for 24 months, their risk of venous thrombosis recurrence is about $40 \%$ lower than before, and there is no increase in bleeding incidence [13]. However, a foreign study on prevention of PICC-related thrombosis found that each method of preventing thrombosis has a protective trend, but no single method can achieve statistical significance alone [3]. Chinese scholar Wan et al. found that it is effective in controlling thrombosis after PICC insertion without significant side effects to give 997 lung cancer patients with PICC a small dose of preventive oral aspirin [14], which is consistent with our findings. Therefore, we believe that Aspirin is safe and effective in being chosen to prevent the formation of venous thrombosis after PICC insertion. However, the research objects in this study are breast cancer patients with PICC, so further research is needed on the relation- 
ship between tumor type and chemotherapy regimen, and between coagulation system and anticoagulant drugs.

\section{Conclusion}

In this study, breast cancer patients with PICC postoperative adjuvant chemotherapy after PICC insertion were enrolled as the research objects, and oral Aspirin enteric-coated tablets were given to prevent the occurrence of venous thrombosis after long-term insertion. The results showed that oral Aspirin can effectively reduce the incidence rate of venous thrombosis in breast cancer patients with chemotherapy after PICC insertion. All patients have no obvious adverse reactions, the cost is low and patients are with good compliance. We believe that it is effective and safe, and it is worthy of clinical application.

\section{Conflicts of Interest}

The authors declare no conflicts of interest regarding the publication of this paper.

\section{References}

[1] Zhao, H.L. (2009) Application and Nursing of PICC in tumor Patients. Journal of Clinical Medicine in Practice, 5, 5.

[2] Chemaly, R.F., de Parres, J.B., Rehm, S.J., et al. (2002) Venous Thrombosis Associated with Peripherally Inserted Central Catheters: A Retrospective Analysis of the Cleveland Clinic Experience. Clinical Infectious Diseases, 34, 1179-1183. https://doi.org/10.1086/339808

[3] Wilson, J.D. and Alred, S.C. (2014) Does Prophylactic Anticoagulation Prevent PICC-Related Upper Extremity Venous Thrombosis? A Case-Control Study. Journal of Infusion Nursing, 37, 381-385. https://doi.org/10.1097/NAN.0000000000000067

[4] Paauw, J.D., Borders, H., Boomstra, S., et al. (2008) The Incidence of PICC Line-Associated Thrombosis with and without the Use of Prophylactic Anticoagulants. Journal of Parenteral and Enteral Nutrition, 32, 443-447. https://doi.org/10.1177/0148607108319801

[5] Han, Q.X. (2012) Improved Sedinggeer Technology PICC Catheter in the Postoperative Chemotheraphy in Breast Cancer Patients. China Practical Medical, 7, 12-13.

[6] Gallina, E.J. (1993) Practical Guide to Chemotherapy Administration for Physicians and Oncology Nurses. Cancer: Principles and Practice of Oncology. 4th Edition, Lippincott, Philadelphia, 2470-2480.

[7] DeSantis, C., Siegel, R., Bandi, P., et al. (2011) Breast Cancer Statistics, 2011. Cancer Journal for Clinicians, 61, 408-418. https://doi.org/10.3322/caac.20134

[8] Agnelli, G. (1997) Venous Thromboembolism and Cancer: A Two-Way Clinical Association. Thrombosis and Haemostasis, 78, 117-120. https://doi.org/10.1055/s-0038-1657512

[9] Zhang, Z., Tang, L., et al. (2017) Progress in the Research on Venous Thromboembolism. Journal of Huazhong University of Science and Technology (Medical Sciences), 37, 811-815.

[10] Guo, D.J. and Hu, D.Y. (2008) Interpretation of the Guidelines of Prevention and 
Treatment of Venous Thromboembolism in Cancer Patients of ASCO. Chinese Journal of Medical Guide, 10, 139-141.

[11] Obialo, C.I., Conner, A.C. and Lebon, L.F. (2003) Maintaining Patency of Tunneled Hemodialysis Catheters: Efficacy of Aspirin Compared to Warfarin. Scandinavian Journal of Urology and Nephrology, 37, 172-176. https://doi.org/10.1080/00365590310008938

[12] Abdul-Rahman, I.S. and AL-Howaish, A.K. (2007) Warfarin versus Aspirin in Preventing Tunneled Hemodialysis Catheter Thrombosis. Hong Kong Journal of Nephrology, 9, 23-30. https://doi.org/10.1016/S1561-5413(07)60005-2

[13] Tufano, A., Guida, A., et al. (2011) Prevention of Venous Thromboembolism in Medical Patients with Thrombocytopenia or with Platelet Dysfunction: A Review of the Literature. Seminars in Thrombosis \& Hemostasis, 37, 267-274.

https://doi.org/10.1055/s-0031-1273090

[14] Wan, G.M., Chen, H.Y., Tang, W.Y., et al. (2013) Effect of Aspirin Intervention on Thrombosis after Application of Peripherally Inserted Central Catheter in Lung Cancer Patients: A Retrospective Observation with 997 Cases. Chinese Journal of Clinical Nutrition, 21, 118-121. 